\title{
3LRM-3 Layer Risk Mitigation Modelling of ICT Software Development Projects
}

\author{
Salma Firdose*, L. Manjunath Rao** \\ * Bharathiar University, Coimbatore, Tamilnadu, India \\ ** Dept. of MCA, Dr. Ambedkar Institute of Technology, Bangalore, India
}

\begin{tabular}{l} 
Article Info \\
\hline Article history: \\
Received Sep 14, 2015 \\
Revised Nov 19, 20 \\
Accepted Dec 3, 2015 \\
\hline Keyword: \\
ICT \\
Risk Analysis \\
Risk Management \\
Risk Mitigation \\
Software Projects
\end{tabular}

Corresponding Author:

Salma Firdose,

Research Scholar, Bharathiar University,

Coimbatore, Tamilnadu, India.

Email: 1snt1@ccu.edu.tw

\begin{abstract}
With the adoption of new technology and quality standards, the software development firms are still encountering the critical issues of risk modelling. With the changing dynamics of customer needs, potential competition has being mushrooming in the global IT markets to relay a new standard of software engineering which has higher capability of sustaining risk. However, till date, it is still theoretical to large extent from research viewpoint. Hence, this paper presents a mathematical model called as 3LRM that is designed with the simple approach keeping in mind the real-time issues of risk factors in software engineering for ICT software development project. The study has also identified requirement volatility as one of the prominent source of risk and hence, the framework intends to identify a risk as well as mitigating the risk to a large extent. The paper is illustrated with some of the simple statistical approaches of random probability.
\end{abstract}

Copyright $(\mathrm{C} 2016$ Institute of Advanced Engineering and Science. All rights reserved.

\section{INTRODUCTION}

The promise of ICT expansion in distressed regions cannot be overstated. Basic elements of ICT [1] have become expected and essentially mandatory resources in developed nations while many parts of the globe remain virtually isolated. There are many factors that have contributed to the current state, and therefore the lack of connectivity and computing resources is not surprising. As the use of ICT in virtually all facets of life in developed nations has continued to grow, the call to introduce the same information technologies into undeveloped regions has become increasingly urgent. Today we are presented with the opportunity to make potentially historic and widespread improvements in the lives of millions by extending the reach of technologies such as broadband networking to drive access to healthcare, e-government, and education resources that would otherwise never reach those who arguably need those most. Despite tremendous progress, the deployment of ICT for development has proven to be a significant challenge. This is due to factors such as high costs of technologies, regional shortages in a skilled labor pool to support deployment, poor physical security and in some cases armed conflict, and others An array of additional economic, political, and social challenges has contributed to the difficulties. In today's scenario, rapid growth of regulations such as international and domestic regulations and modern world market are appropriate in the nation where activities of businesses are conducted to make risk management a prerequisite for stable business. The unavoidable part of every business activity i.e. risks within the IT system are also a part of management and risk management process. Risk treatment within the IT system is predominantly valuable as because of the vibrant nature of intimidation and accelerated development of technology [2]. The lack of ability of timely appreciation of all scenarios which brings threat to IT systems can effect in unproductive and expensive security procedures. An important characteristic in the deployment of any information system 
is reliability. There is a need for resilient ICT. Stated another way, maximizing resiliency in ICT will contribute to maximizing return on ICT investment as a reliable system that will meet expectations in delivering critical services more closely. Today risk is generally increasing due to the challenges of globalization, technological complexity, increased technical and process interdependencies, and other factors. The proposed paper discusses about such new model. The paper presents a mathematical model of risk identification \& mitigation. Section-1.1 discusses about the background of the study discussing an impact of the risk factors on the standard models followed by problem identification discussion on Section-2 discusses about Result Discussion. Finally, some concluding remarks are made in Section-3.

\subsection{Background}

It is very important to chose a right model for mitigating requirement volatility in the field of risk scheduling process, as already resources and cost are deployed in the risk scheduling process and if in appropriate model is selected, then, other than slicing down the cost and schedule, it can increment it. It has been seen that the structure, categorization and vocabulary of parameters and metrics applicable to software quality management have been derived or extracted from the ISO 9126-3 and the subsequent 25000:2005 quality model [3] [4] [5]. In the area of Information technology, software process improvement is a major conception and it has actually a potential of mitigating various software project risk scheduling in terms of requirement volatility prominently [6] [7]. Among the entire quality standards, the frequently used standards are ISO standards, Total Quality Management (TQM), Kaizen, and Capability Maturity Model (CMM). Majority of the MNC organization is seen to practice CMM which is designed by Software Engineering Institute (SEI). CMMI (Capability Maturity Model Integration) which facilitates organizations to gauge their "maturity" on a scale of one to five which is represented as initial, repeatable, defined, managed and optimizing in working on software engineering. Enhancement is accomplished by action policy for neglected areas. Requirement specification is very frequently is never predictable as it is uniformly changing along with the project development is in progress. Such types of organizations in this level do not have an efficient project management processes including risk scheduling process and will not support appropriate risk scheduling.

The fundamental of the project management processes are firmly established in the second level where managing and planning of new requirement is based on previously maintained records and level of accomplishments in past will be repeated. So this level can assure more error free results compared to previous level in risk scheduling [9] [10]. This level lacks the support of organized and documented plan for risk management though first model can be mechanized in this stage. Along a distinction with previous level, this level can let the organization to use second model as they executes requirement management process here. Software Product Engineering of level 2 recommends that the requirement documents be managed through version-control and change control practices, this can help in calculating metrics which are required for second model to avoid the most frequent problem of requirement volatility.

It can be evenly said that a company with a distinct set of quality standards for risk management processes and provides mechanism to support for executing such quality standards can be considered as more mature than a company with only informal standard definitions. For overcoming the risk issues like requirement volatility, any undocumented risk related parameters cannot be accounted to mitigate risks. The development scenario must allows proper documentation of all the steps of the requirement understanding from the client as well as all the formal communication for requirements with client and the development team should be properly analyzed and documented to avoid requirement change in the progress stage of the ICT software project development.

\subsection{The Problem}

As the range and complexity of computer applications have grown, the cost of software development has become the major expense of computer-based systems. Research shows that in private industry as well as in government environments, schedule and cost overruns are tragically common. Despite improvements in tools and methodologies, there is little evidence of success in improving the process of moving from the concept to the product, and little progress has been made in managing software development projects. Research shows that 45 percent of all the causes for delayed software deliveries are related to organizational issues. Despite the recent improvements introduced in software processes and automated tools, risk assessment for software projects remains an unstructured problem dependent on human expertise. The acquisition and development communities, both governmental and industrial, lack systematic ways of identifying, communicating and resolving technical uncertainty. Solving the risk assessment problem with indicators measured in the early phases would constitute a great benefit to software engineering. In these early phases, changes can be made with the least impact on the budget and schedule. Part of the problem is misinterpreting the importance of risk management. A second source of problems in risk management is the 
lack of tools. The main reason for this lack of tools is that risk assessment is apparently an unstructured problem. To systematize unstructured problems it is necessary to define structured processes. Structured processes involve routine and repetitive problems for which a standard solution exists. Unstructured processes require decision-making based on a three-phase method (intelligence, design, choice) [12] [13]. An unstructured problem is one in which none of the three phases is structured. Current approaches to risk management are highly sensitive to manager's perceptions and preferences, which are difficult to represent by an algorithm. Depending on the decision-makers attitude towards risk, he or she can decide early with little information, or can postpone the decision, gaining time to obtain more information, but losing some control. A third source of risk management problems is the confusion created by the informal use of terms. Often, the software engineering community (and most parts of the project management community uses the term "risk" casually. This term is often used to describe different concepts. It is erroneously used as a synonym of "uncertainty" and "threat" [14] [15]. Generally, software risk is viewed as a measure of the likelihood of an unsatisfactory outcome and a loss affecting the software from different points of view: project, process, and product. However, this definition of risk is misleading because it confounds the concepts of risk and uncertainty. In general, most parts of decision-making in software processes are under uncertainty rather than under risk. Uncertainty is a situation in which the probability distribution for the possible outcomes is not known. We address the issue of risk assessment by estimating the probability distribution for the possible outcomes of a project, based on observed values of metrics that can be measured early in the process. The metrics were chosen based on a causal analysis to identify the most important threats and a statistical analysis to choose the shape of the probability distribution and relate its parameters to readily measurable metrics.

\subsection{The Proposed Solution}

The prime goal of the proposed study is to present a mathematical modelling of risk mitigation schema considering the real-time project management issues of ICT software project development. The study is done from software engineering viewpoint. The proposed model presents 3 layer of mitigation approach in risk management and hence is termed as 3LRM Model, which mean 3-layer of Risk Mitigation Model. The discussions on the 3 individual layers are stated below:

\subsubsection{Layer-1 Approach}

This is preliminary layer under discussion which is basically a framework for identifying ICT risk involved in project development pertaining to software engineering. Primarily is this layer, projects and environment are rendered very much dissimilar from the instance when the work is presented. Secondarily, technology as well as organization structure is assumed to have enormously undergone changed. That will be the reason that an analysis to discover universal requirement volatility record in recent development has been performed with the certain objectives. First goal of this layer is to find the parameter, which project leaders, distinguishes as risk and also identifying that which factors are more vital in view of project leaders. Second is to classify risk parameters in a way that common improvement policy can be used for each classification. Robust association between importance of mitigating imprecise requirement and apparent intensity of managing was observed very crucial; as even with high risk, low apparent intensity of project risk scheduling permits a few for encountering that ICT project risk. This is a very simplistic and implementable layer that will substantially concentrates on scheduling risk related to a very vague or imprecise requirements only are highly qualified professionals. The work has an importance of scheduling risk and various intensity of managing a project those technical leaders has. Basically, it recommends an analytical framework for scheduling risk with respect to requirement volatility for diversified types of other risk classifications with Strategical solution for each classification of risk.

\subsubsection{Layer-2 Approach}

This is the second layer under discussion which is about a format risk assessment framework for analyzing software engineering. In case of traditional software development, ICT project requirement frequently changes as development proceeds. In fact the surfacing viewpoint has now turned into the standard in ICT project risk management and as such a query of schedule and expenditure overrun becomes serious where the solution lies in executing appropriate risk scheduling. Recently early requirement volatility is an amorphous problem, which depends on individual human judgments and unreasonable hypothesis such as, not altering necessities and work breakdown structure. To highlight such issues, risk assessment has to be more ordered, efficient, and goal oriented. There is no consideration of requirement volatility in the existing models, which is one of the significant parameter in ICT projects. Some other important parameters were also not considered like complexity of the project, skill gap analysis, human resources, and efficiency of the project team involved. This layer is basically formulated to analyze and address all these critical issues. This 
layer is based on requirement volatility, complexity and efficiency. The Requirement volatility $(\alpha)$ can be estimated by summing up Requirement Birth Rate $(\beta)$ and Requirement Death Rate $(\gamma)$ of the ICT software project, and can be mathematically represented as,

$$
\begin{aligned}
& \alpha=\beta+\gamma \\
& \alpha=\left\{\frac{\sigma}{\omega} \cdot 100\right\}+\left\{\frac{\eta}{\omega} \cdot 100\right\}
\end{aligned}
$$

Where, $\sigma$ represents number of new requirements, $\eta$ represents number of requirements deleted, and $\omega$ represents gross number of requirements. As the proposed study revolves around software engineering, where understandability, learnability, and operability are some of the prime attributes in modelling, hence, its complexity-factor of the risk are empirically represented as:

$$
C_{F}=\delta_{1}+\delta_{2}+\delta_{3}
$$

Where,

$\delta_{1}=$ No. of state machine

$\delta_{2}=$ No. of data connection between operator

$\delta_{3}=$ No. of abstract data type required for the system

Finally, the system will compute productivity of the layer-2 approach by using eq (6)

Productivity $=$ Direct Labor Time / Idle Time

As per the model, a random variable $x$ is said to have a random probability distribution with the parameter $\Omega_{1}, \Omega_{2}$ and $\Omega_{3}$ (with $\alpha>0, \beta>0$ ) probability distribution function (PDF) and cumulative distribution function (CDF) are of $x$ are respectively.

$$
\begin{aligned}
& f\left(x: \Omega_{1}, \Omega_{2}, \Omega_{3}\right)=\left\{\begin{array}{c}
0 \\
\left(\Omega_{1} / \Omega_{2}\right)^{\Omega_{1}}\left(x-\Omega_{3}\right)^{\Omega_{1}-1} \exp \left(\left(\left(x-\Omega_{3}\right) / \Omega_{2}\right)^{\Omega_{1}}\right)
\end{array}\right. \\
& f\left(x: \Omega_{1}, \Omega_{2}, \Omega_{3}\right)=\left\{\begin{array}{c}
0 \\
\left(1-\exp \left(\left(x-\Omega_{3}\right) / \Omega_{2}\right)^{\Omega_{1}}\right)
\end{array}\right.
\end{aligned}
$$

The above equations (7) and (8) shows PDF and CDF where the first condition is valid for $(\mathrm{x}<\Omega 3$ ), while the second condition is valid for $(\mathrm{x} \Omega 3)$. The random variable under study, $\mathrm{x}$ can be interpreted as development time in our context. The shape parameter $\Omega 1$ controls the skew of the PDF, which is not symmetric. We found that this is mostly related to the productivity of the organization. The scale parameter $\Omega 2$ stretches or compresses the graph in the $\mathrm{x}$ direction. It can be seen that this parameter is related to the productivity $(\mathrm{P})$, requirements volatility $(\alpha)$, and complexity $(\mathrm{CF})$. The shifting parameter $\Omega 3$ shifts the origin of the curves to the right. This model is perfectly suited for projects, which are evolutionary in nature. The results of the model can be validated by COCOMO [16] [17].

\subsubsection{Layer-3 Approach}

The third layer is about software risk assessment which highlights an issue of unsuccessful failure for designing product within specified time frame and allocated cost. It also discusses another problem which either the product is in accordance to client's condition along with their satisfaction level. It assumes 9 parameters which give birth of diversified category of requirement volatility in the stage of near completion of the ICT project and will then definitely influence the defined project development timetable, quality along with cost involved. Parameters are product's complexity, human resources involvement in the ICT project, targets for reliability, requirement of product, cost estimation methodology, process monitoring, software development life cycle adopted, software usability and project development technology. It associates the Client's Feedback Index with the project wholesome risk and offers following implications. For example if Client's Feedback Index is less than 5, than we can call that project is not acceptable, if it is between 5 and 10 then the project may be ended with far-reaching schedule and substandard. If it is between 15 and 10 then project completed within due specified dates in pre-allocated cost and as per terms and specifications of the 
client. For evaluation the project manager answers the questionnaire, recommended by this model, according to the type of the project. Estimate the statistical value connected with the selected choice. Evaluate the normalized figure for each of the nine risk parameters by appending the statistical scores of the question project manager tried and by dividing the total number of query tried. Then estimate the normalized requirement volatility for the project by using following formula for Normalized Requirement Volatility

$$
\alpha_{\text {norm }}=\frac{\alpha-\alpha_{\min }}{\alpha_{\max }-\alpha_{\min }}
$$

It gives an objective numerical figure for nine areas of the project and also recommended an empirical formula to evaluate for entire project using the nine-risk parameter of the project. It can be estimated. It associates the risk value with the customer feedback index, which is an indication of the customer feedback that relates to the standard of the product. It has minimum empirical score when it comes to associate risk value with client's feedback index as results are based on same categories of ICT projects.

The model is integrated and evaluated for the purpose of testing its efficiency. A survey is conducted online with different level of quality standards in order to recognize more serious issues of requirement volatility. The findings was intended to conclude which framework is the best suited for companies encountering failures in maintaining risk scheduling schemes. The target of the evaluation by number of project leaders is estimate the risk identification which could possibly have serious impact on ICT project schedules, expenditure etc. The next important phase of the 3LRM Model is to formulate strategies for major risk identification. There is a diversified outcome of the survey where the similar risk is evaluated by different participants with varied prioritization. A nontrivial strategy is adopted to understand the seriousness of risk involved. A Pseudorandom weight of 1,2, and 3 is assigned to risk with 3rd, 2nd and 1st priority order. Therefore, if the same risk is found in varied order than it will possess the cumulative risk weightage value as estimated by product of risk weight value with number of human resource selected at $1 \mathrm{st}$ priority which is again summed up with risk weight value with number of human resources at the 2nd priority and it continues. There for final risk weight was normalized. The evaluation can be conducted by estimating rank of risk is:

$$
\mathrm{R}_{\mathrm{rank}}=\left(\psi_{1} \cdot \lambda_{1}\right)+\left(\psi_{2} \cdot \lambda_{2}\right)+\left(\psi_{3} \cdot \lambda_{3}\right)
$$

Where, $(\psi 1 . \lambda 1),(\psi 2 . \lambda 2)$, and $(\psi 3 . \lambda 3)$ are the set of risk values $(\psi)$ and frequency $(\lambda)$ of the risk mapping with 1st, 2nd, and 3rd priority. The model proposes that project leaders should first accept those risk factors which have comparatively maximum relative significance and have greater professed score of control. It is then feasible to design risk encountering polices depending on the nature of risk can be developed. Frequency of risks in 1st priority cannot be efficiently controlled by project leaders. Therefore, formal step for executing the framework will be primarily to estimate the risk ranking according to ICT software, secondarily to classify it in the highlighted model, and finally to relate certain prescribed method to those risks factors which lie in third column.

\section{RESULT AND DISCUSSION}

The above discussed model is evaluated in diversified companies online which follows different quality standards and practices. A qualitative technique of standard survey has been conducted to validate the effectiveness of layer-based approach and third layer approach. The outcomes of the survey were subjected to quantitative analysis to find that the proposed model do produce consistent measures of risk schedule for these types of project development. But it does not specify that this framework is not effectual as certain implications found in this framework indicate that the framework can be appropriate on traditional ICT project development. It has been seen that in order to generate Weibull curve the factor $\Omega_{2}$ has to be much higher than $\Omega_{3}$, and in context of this framework, it is only feasible when requirement volatility is much greater which is an intrinsic characteristics the environment of traditional ICT project development. Based on this fact, it can be stated that this framework would be efficient for traditional ICT project development. The evaluation has also identified certain issues with this framework. However, this will be the focus of our future work. The framework was found to be complicated, estimating factors required by the model like requirement volatility and risk scheduling will require proper elucidation of various multifaceted factors, which if estimated inappropriately will result in high cost expenditure. The time for execution is found to be high, while the project leaders normally don't have much time. No way, it is appropriate for ICT software project development other than traditional type. In short, this framework can only be used for frequent 
changes in requirements for ICT project of complex type with some targeted evaluation required. The framework of first layer is on evaluating the major risk and then analyzing the ICT project subjected to those risks. It assumes that if the risk is raised by project leaders than they can take up appropriate risk scheduling measures to encounter it. In case the project leaders have maximum score of scheduling the risk and that risk has comparatively greater significance than a project leader should assume those risk first for encounter strategy. As there are very few project leaders can do for those risks factors, which are not in their control. The other two layers (second and third) did not assume this factor. All the three layers other than second layer are based on targeted evaluation. But the problem with second layer is it's difficult for estimating the factors which is needed by the framework, besides it is appropriate for ICT software projects. In comparison to derived model with first layer, it is observed that this framework has significant risk factors which are not described by first layer. Therefore, the proposed study furnishes a highly optimized outcome for identification of the uncertainty that is mainly interpreted as a risk factor. The proposed methodology adopted for the study is based on the fact that it is not possible to ascertain and mitigate complete risk factor, but it is possible to model a framework on a given constraint of case study and its associated resources to measure uncertainty factor in the software development methodologies. The proposed study is evaluated wityh respect to normalized requirement volatility $(\alpha)$ as the performance parameters along with Requirement Birth Rate $(\beta)$ and Requirement Death Rate $(\gamma)$ for enhanced preciseness in the outcomes.

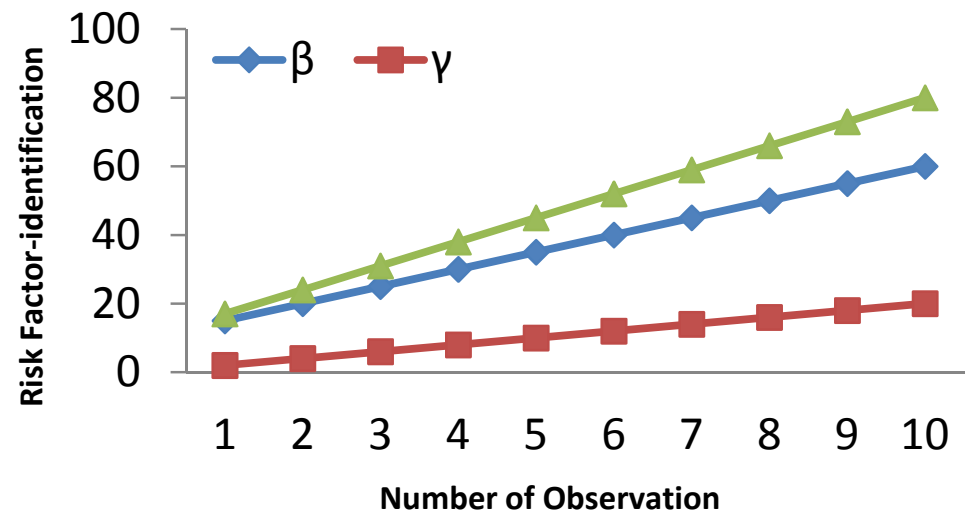

Figure 2. Risk Factor Identification

The representation of the risk factor on the increasing number of observation is shown in Figure 2. The outcome clearly exhibits that there are three curves considered for comparative analysis i.e. requirement volatility $(\alpha)$, Requirement Birth Rate $(\beta)$, and Requirement Death Rate $(\gamma)$ for enhanced preciseness in the outcomes. Owing to the optimization principle incorporated in the proposed system, the system continuously seeks the belite outcomes of mitigating uncertainty measures. This is the prime reason for requirement death rate is found to be diminished for the given example in Figure 2.

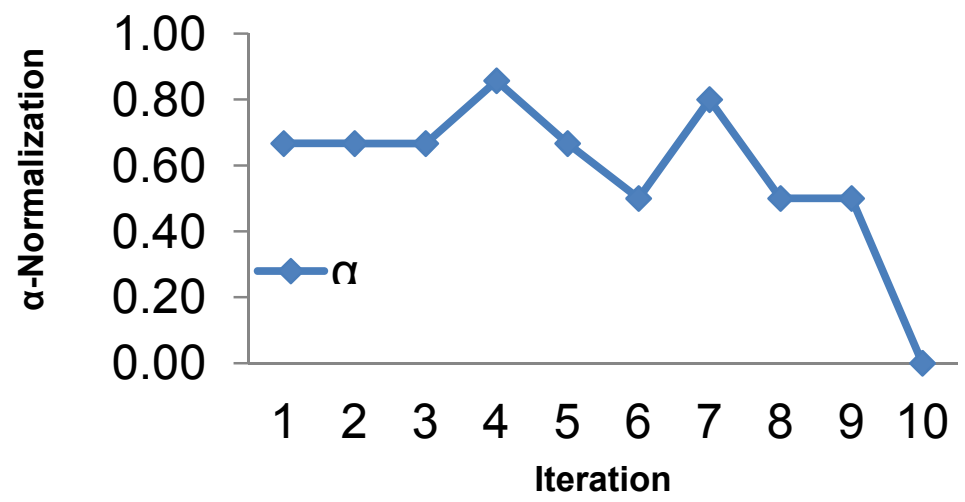

Figure 3. $\alpha$-Normalization curve 
After performing empirical evaluation of the proposed mathematical modelling, the score of effectiveness of the proposed system is evaluated by observing i) potential of model for identifying risk factor and ii) $\alpha$ normalization curve. Considering 10 levels of observations using random probability distribution model, the outcome shows potential risk identification capabilities as exhibited in Figure 2 that shows risk-identification $(\alpha)$ is quite high as compared to requirement birth rate and requirement death rate. Similarly, the model also shows better gradient descent for risk normalization using equation (7). Hence, it can be said that the proposed model has better capabilities for identifying risk (Figure 2) and mitigating risk (Figure 3). The extensive investigational analysis has been conducted in previous paper [18].

\section{CONCLUSION}

The proposed study presents a mathematical model that has the capability of identifying the risk as well as mitigating the risk. It is also helpful to deploy the effective framework which is very expandable and scalable inspite of wasting resources for mechanizing new framework. Based on the analyzation of the frameworks discussed, a framework has been formulated for companies, which comes under the different levels of quality standards. Various risk scheduling process are the major local organizational factor that can impact on the effectiveness of the framework, so it is imperative to choose the appropriate evaluation framework by associating the quality standards of an organization with the framework. In order to facilitate project leader for adopting the right framework, the described framework has correlated with different quality standards. The existing research on risk scheduling and requirement volatility has enormous feasibility to rise in multi-directions.

\section{REFERENCES}

[1] M. Lazzaroni, "Reliability Engineering: Basic Concepts and Applications in ICT", Springer Science \& Business Media. Technology \& Engineering, pp. 176, 2011.

[2] J.C. Cummings, "Modelling and simulation to support risk management in complex environment", Retreived from http://www.tisp.org/tisp/file/CUMMINGS_Paper_Mod-Sim-Risk-Mgmt_Paper.pdf, 2014.

[3] J. Münch, O. Armbrust, M. Soto, M. Kowalczyk, "Software Process Définition and Improvement", Distance and International Studies Center. Springer, 2012.

[4] N.O. Gass, "Deployment of Radio Frequency Identification (RFID) in the oil and gas industry Part 2 Architecture and integration", Norwegian Oil and Gas Guideline 112, Part 2 approved by: Norwegian Oil and Gas's Director General, 2012.

[5] A.M.A. Khouri, "Using Quality Models to Evaluate National ID Systems: the Case of the UAE", International Journal of Social Sciences. Vol. 1 No. 2, 2007.

[6] N. Nurmuliani, D. Zowghi, and S.P. Williams, "Requirements volatility and its impact on change effort: evidencebased research in software development projects", Proceedings of the Eleventh Australian Workshop on Requirements Engineering, 2006.

[7] R. Thakurta, S. Dasgupta, "Using System Dynamics Modeling to Investigate the Impact of Resource Management Policies on Project Quality Assurance under Requirement Volatility", International Journal of Information Processing and Management, Vol.2, No. 3, 2011.

[8] P.K. Dey, "Project risk management: a combined analytic hierarchy process and decision tree approach", Cost Engineering, Vol. 44(3), pp. 13-27, 2002.

[9] HY, C., X. Liang, and C. Song, Z, "Project Duration Risk Transmission and Control based on CCPM.Research", Journal of Applied Sciences, Engineering and Technology, Vol. 7(3), pp. 619-624, 2014

[10] S.V. Bharathi, R. Raman, and D. Pramod, "A FPN Based Risk Assessment Model for ERP Implementation in Small and Medium Enterprises", Middle-East Journal of Scientific Research, Vol. 19 (6), pp.747-759, 2014.

[11] A.K. Madan, M.S. Ranganath, "Multiple Criteria Decision Making Techniques in Manufacturing Industries-A Review Study with the Application of Fuzzy", International Conference of Advance Research and Innovation, 2014

[12] A. Ugale, S.D. Kamble, P. Mishra, P, "Classification of Dataset Using Business Intelligence for IT Support Services", International Journal of Research in Advent Technology, Vol. 2, No.2, E-ISSN: 2321-9637, 2014.

[13] D. Hillson, "Managing Overall Project Risk", Project Management Institure, 2014.

[14] A.A. Khan, R. Gowda, "Risk and Uncertainty in Business", IRACST - International Journal of Commerce, Business and Management (IJCBM), ISSN: 2319-2828 Vol. 3, No. 1, 2014.

[15] K. Garg, P. Kaur, S.Kapoor, S. Narula, "Enhancement in COCOMO Model Using Function Point Analysis to Increase Effort Estimation", International Journal of Computer Science and Mobile Computing, Vol. 3, Issue. 6, pp. 265-572, 2014.

[16] I.K. Walia, and M. Kaur, M, "Combining COCOMO and use case for better effort estimation", International Journal of Current Engineering and Technology, Vol. 4, No. 2, 2014.

[17] A. Kinra, "A Fuzzy Based Model for Software Quality Estimation Using Risk Parameter Assessment", International Journal of Computer Science and Mobile Computing, Vol. 3, Issue. 3, pp. 807-814, 2014. 
[18] S. Firdose, and L.M. Rao, "Investigational Analysis of existing Risk Management Models and Prior Research Contribution: A Review", International Journal of Advanced Research in Computer Science and Software Engineering. Vol. 4, Issue. 12, pp. 882- 889, 2014

\section{BIOGRAPHIES OF AUTHORS}

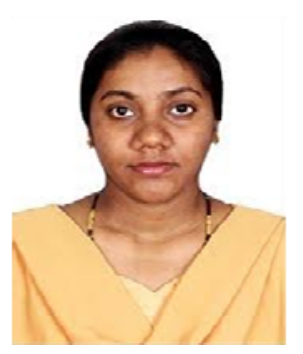

Salma Firdose did her Bachelor of Science from Bangalore University from 2000 to 2003. In 2003 received the Bachelor degree. She Studied Masters of Computer Application from Bangalore University from 2003 to 2006 and was awarded masters in the same year.In 2007 to 2009 did Master of Philosophy from Bharathiar Universiy, Coimbatore. Now she is a Ph.D. student 3rd year of CSE at Bharathiar University, Coimbatore, India. She worked as lecturer for 6 years at Bangalore, India and 2 years in abroad. Now currently working at Bangalore, India

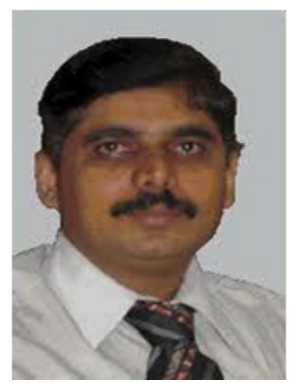

Dr. L. Manjunatha Rao is working as Professor and Head, Department of MCA, Dr. AIT, Bangalore. He has got 25 years of teaching experience. He did his Bachelor of Science from Bangalore University in the year 1990. He Studied Masters of Computer Application from Madhurai Kamaraj University and was awarded in the year 1999. In 2002 did Master of Philosophy from Mononmanium Sundaranar University. He has awarded Ph.D from Vinayaka Mission University, Tamil Nadu. He has published research papers in both national and international Journals and has authored 2 textbooks. 